\title{
Critical Review: An Evaluation of Nitrous Oxide Analgesia During Transcutaneous Pacing by Kaplan, Heller, McPherson, and Paris
}

\author{
Michael Moles, MB, BS, FFARCS
}

In the paper, Evaluation of Nitrous Oxide Analgesia during Transcutaneous Pacing, by Kaplan et al., which appeared in the second issue of Prehospital and Disaster Medicine, there are several points made by the authors which require careful consideration before they are put into effect by the corps of EMTs and paramedics.

The search continues for a solution to the outstanding problem of discomfort and pain related to transcutaneous pacing (TCP), which presently constitutes a constraint on the application of TCP as a life support intervention. This paper reports a laboratory evaluation of the inhalation of 50:50 $\mathrm{N}_{2} \mathrm{O} / \mathrm{O}_{2}\left(\mathrm{~N}_{2} \mathrm{O}\right)$ as an analgesic, and proposes extrapolation of the results into the prehospital environment.

The study is important on two counts. First, it utilizes methods taken from analgesimetry, a technique widely applied but often with defective rigor in anesthesia, obstetrics, dentistry, and clinical pharmacology; and, second, by virtue of its position in a cohort of progressive studies, it may serve as a potential template for future investigations. The paper merits critical consideration on both counts. This commentary first will address general considerations and then specific aspects, in manuscript order.

The study exhibits, and the paper concedes, overt omissions in the rigor of design; firstly, in the application of the reference analgesimetry model and secondly, in the logic of the extrapolation to the clinical setting. In addition, however, there are covert omissions in the rigor of method of both data acquisition and processing. The entire study would be enhanced by the provision first of more explicit information on rationale, criteria, and technique of management of material, method, procedure, and protocol, which would both improve rigor and facilitate audit and evaluation of the study. In addition, if more detailed data were presented, it would help to identify internal errors and facilitate analysis and validation of the results.

The abstract and introduction are excellent. They provide a concise and lucid account of the investigative hypothesis and protocol. The methods invite consideration of the following comments, cataloguing errors, and omissions.

There is no statement of approval from ethical and experimental committees; perhaps this was implicit in the form of consent.

No rationale or criteria are given for the selection of the pacemakerelectrode combination in each subject; this is germane to the exclusion of correlation bias. There is no specific evidence of exclusion of confound-

Prince Philip Dental Hospital, University of Hong Kong 
ing variables, incidental to each subject but capable of differentially affecting pain threshold or perception between paired experiments in this small sample, for example: medication, including psychotropics; fasting; food and alcohol intake; exercise; circadian endocrine/endorphin variations, e.g., menstruation; and diurnal endorphin variations, i.e., mandating the use of fixed time frames. Unblinded exposure in the $\mathrm{N}_{2} \mathrm{O}$ trials, by use of a mask demandvalve system introduces a placebotype bias; this error could and should be quantified in a comparative trial blinded by use of cylinder medical air delivered through an identical system. Prior exposure to $\mathrm{N}_{2} \mathrm{O}$, providing previous knowledge of effect, unequivocally compounds this error with a second conditioned bias favoring $\mathrm{N}_{2} \mathrm{O}$; this error also might be quantified by a similar trial blinded for both air and $\mathrm{N}_{2} \mathrm{O}$ with, perhaps, mercaptan.

Random protocols determined the sequence of experimental pairs, air, or $\mathrm{N}_{2} \mathrm{O}$ first; but pacemaker threshold was determined after inhalation of $\mathrm{N}_{2} \mathrm{O}$ (or air). The effect of $\mathrm{N}_{2} \mathrm{O}$ on TVP threshold is known and minimal but that on TCP threshold is not; if there is an effect there might be a further bias.

The rationale given for selection of a five-minute "induction time" is insufficient. Pharmacokinetically, for $\mathrm{N}_{2} \mathrm{O}$, the rate of equilibration of alveolar and inspired gas fractional concentration, partial pressure, and analgesia is determined by alveolar ventilation, cardiac output (and shunt fraction); was ventilation "controlled"? Cardiac output would be raised both by pacing and the experimental model; this would slow the rate of "induction."

Pharmacodynamically, $\mathrm{N}_{2} \mathrm{O}$ itself affects ventilation and cardiac output but the effect is still dynamic at five minutes. In obstetrics, analgesia is obtained in 90 seconds. No rationale is given for the selection of the 30 seconds TCP "challenge time." Was this dictated by empirical, ethical, or cardiological constraints? If not, why was extended TCP tolerance time not subjected to measurement rather than subjective "guesstimation," and extended to a "median prehospital run time," as the authors suggest?

Criteria for premature termination of pacing are omitted. The range given for PVAS (pain visual analog score) extends only to 8 which seems paradoxical. The last sentence of the methods section seems far from exact! The results are presented discursively, nonalgorithmically, and, despite the small sample, with only summary information, omitting supportive detailed and tabulated data, thus frustrating both internal and external correlation analysis. There is an obfuscating lack of coherence of treatment between the methods and results, for example: "Electrical capture was documented for each of the room air trials," omitting comment on capture verification in the $\mathrm{N}_{2} \mathrm{O}$ trials; or is this at variance with the randomization protocol? "Prolongation time" appears in the results without reference or definition.

Such data, as are given, merit comment. Of the 18 subjects remaining after exclusion, 15 expressed subjective preference in favor of $\mathrm{N}_{2} \mathrm{O}$, for which significance is omitted. The only significant difference between the air and the $\mathrm{N}_{2} \mathrm{O}$ trial group is at $p<0.05$. The pacing time (TCP tolerance time) trials report means at 22.4 and 23.8 seconds, with respective standard deviation of 10.2 and 9.6. These data are not normally distributed and the $t$ test is invalid. In addition, a Chi-square test is invalid when cells contain very low counts, as appears to be the case here.

In the subset of six subjects, favoring $\mathrm{N}_{2} \mathrm{O}$ but not achieving a TCP tolerance time of 30 seconds, large variability was noted, reproducing scatter noted in one pilot study (reference 10). Data are omitted on scatter for the other subsets. Capture threshold is reported as $107 \pm 37 \mathrm{~mA}$ (sic? s.d.), implying a range of at least 70 to $144 \mathrm{~mA}$. Insufficient data are provided to determine correlation between threshold and either discomfort rating or TCP tolerance time.

These laboratory results must be considered equivocal and indeterminate. Thus, subject to the considerations above, the study valuably could be repeated, augmented, and extended. In the laboratory and the field, non-invasive saturation oximetry measurements should be incorporated. Paradoxically, in the critically ill, hypothetical consideration of $\mathrm{N}_{2} \mathrm{O}$ kinetics and dynamics favor both rapid onset and synergy with other modes of analgesia.

Finally, a question: if pacers with a pulse duration of $20 \mathrm{msec}$ consistently evoke more pain than a pacer with a pulse duration (PD) of $40 \mathrm{msec}$ (not used in this study and operating in different mode), then, subject to technical and clinical feasibility, might this not suggest an alternative vector for research and development? 